\title{
General Discussion of Accretion Disks
}

\author{
Gurzadyan, Vahagn G.
}

Department of Theoretical Physics, Yerevan Physics Institute, Yerevan, 375036, Armenia.e-mail: gurzadya@lxz.yerphil.am

Even 25 years after the Shakura-Sunyaev seminal paper on the $\alpha$-disk, we cannot claim that we have a reliable theory of accretion disks in galactic nuclei. Why? Because the problem is extremely complicated, it is essentially nonlinear and contains a number of parameters (i.e. is many-dimensional). The key point is whether it is possible to determine the magneto-hydrodynamical viscosity self-consistently, i.e. as a function of parameters of the disk - the temperature, matter and radiation densities, magnetic field, radius, etc., both in the radiation dominated and matter dominated regions. Another class of fundamental problems concerns the stability of the disk; Krolik mentioned only one instability - in the radiation dominated region, but there are many other types of instabilities which are quite sensitive to the physical conditions in the disk, for example, to the anisotropy of the ion pressure in the outer regions and possible electron-positron pair production near the inner edge of the disk. The other problems include those of the radiative transfer within the disk in various conditions, Comptonization of the outgoing radiation, radiation reflections by the desk, etc. Therefore it is not suprising that one can 'explain' almost whatever he wants - spectra, variability, jets, wind, etc., by proper fit of the 'free' (which are never free) parameters and ignoring the instabilities and so on.

Thus, both the apparent coincidence and disagreement (as claimed here by Sulentic) of certain observational data with the $\alpha$-disk predictions, are equally not sufficient to conclude the existence or absence of accretion disks in those objects. Moreover, even the disk-type structures directly observed in certain galactic nuclei (they are extremely impressive!) cannot be interpreted uniquely as the accretion disks we are speaking about. Not every Keplerian disk has to be an accretion disk.

There is no hope that a complete self-consistent theory of turbulent accretion disks can be created in the near future. Thus, I see the following way for further development of the theory. Any nonlinear many-dimensional dynamical system (in particular case, given via a system of differential equations) due to unstable (chaotic) regions of the phase space can have both - properties very sensitive to the control parameters and boundary conditions, and those robust to the latter. Therefore it will be of particular importance to look for corresponding formulations of the disk problem and the search for their robust properties. Only those robust properties can make sense to be compared with the observations. An example of such a mathematically correct formulation of the problem can be the one reported by Fridman at this meeting.

To be brief, among many other problems, I mention only the following inevitable source of accreting matter which strangely was less discussed during present meeting - the tidal disruption of stars within the Roche lobe of the 
massive centre. The crucial point here is that, the character of accretion depends not only on the mass of the centre but also on the dynamical parameters of the surrounding stellar system (Nature, 280, 214, 1979). Given the present possibility of accurate determination of the parameters of the stellar systems surrounding the AGN engines, this effect has to be considered quite seriously.

Thus, it seems there is still an essential way to go both from the present toy disk models to more complex theories, as well as in the cautious and balanced interpretation of the ever increasing flow of excellent observations, in order to understand properly the basics of the AGN mystery. 